\title{
Axial Wave Reflection and Transmission in Stepped Nanorods Using Doublet Mechanics Theory
}

\author{
Metin Aydogdu, ${ }^{*}$ Ufuk Gul \\ Department of Mechanical Engineering, Trakya University, Turkey
}

\begin{abstract}
A numerical investigation of the reflection and transmission of axial waves at stepped nanorods is presented. The scale dependent doublet mechanics theory is used in the analysis. The main difference of the doublet mechanics from other scale dependent models (stress gradient, strain gradient and couple stress theories) is its direct dependence to the micro/nano structure of the solid. Scale parameter is directly related to atomic structure of the material in doublet mechanics theory and it is assumed as carbon-carbon bond length in the present study. However, identification of scale parameters in other scale dependent theories is difficult compared to doublet mechanics theory. Governing equations of stepped nanorods are derived in the framework of doublet mechanics using the Hamilton Principle. The numerical results predicted by doublet mechanics are shown and compared with the classical elasticity.
\end{abstract}

\section{Introduction}

In the last two decades, carbon nanotubes have been attractive due to their excellent mechanical, chemical and thermal properties so they have been used for designing of new nano-electronics devices, nanocomposites, nano electro-mechanical systems (NEMS). The determination of transmission and reflection coefficients in stepped nanorods is also a crucial problem while studying the structure-borne sound, propagation and radiation in nanostructures. Cramer and Heckl [1] have studied the reflection and transmission coefficients at the discontinuity cross section using the one dimensional wave equation. For larger wavelengths, these coefficients have been computed by Hagedorn and Seemann [2] in rods and beams considering the classical rod and beam theory. Love's theory which takes into account the kinetic energy due to lateral motion was used in order to calculate the transmission and reflection coefficients of a stepped rod by Seemann [3]. He compared the obtained results with the FEM solution and 3-dimensional theory of elasticity for validation. Mace [4] derived the transmission and reflection matrices for the cases of a point support and a sudden change in cross-section of the Euler-Bernoulli beams. The results were given for the free and forced vibration cases. Wave propagation of tunable fluid-filled beams has been investigated considering the wave reflection and transmission at a change in cross-section by Harland et al. [5]. Also, some studies which are related to wave reflection and transmission in Timoshenko beams have been examined in [6-8]. Recently, wave reflection and transmission of carbon nanotubes have been analysed by using finite difference time domain method [9]. The main aim of these papers is to derive the reflection and transmission matrices under the discontinuities in structures. This provides a concise and systematic approach for analysis of the structures.

Classical continuum models are not suitable for modelling of nano scale structures due to their size effects and molecular dynamics simulation has some difficulty while computing the nano-scaled structures. So, some different size dependent continuum models have been proposed in the open literature such as strain and stress type gradient models, modified couple stress theory, peridynamics. They have been used in static and dynamic analysis of nanostructures [10-12]. The main purpose of these theories was the consideration of the internal length scale parameter of the material while modelling their mechanical responses. An alternative micro mechanics scale dependent theory, DM was proposed by Granik [13]. Granik and Ferrari [14] and Ferrari et al. [15] have used DM theory in granular materials modelling. DM theory is not a phenomenological theory in contrast to other size dependent continuum models. In DM theory, atomic distance of elastic material is directly used as an intrinsic length scale parameter. This makes DM model more physical compared to other size-dependent continuum models. Also the approximation of the micro structure of the solid can be controlled by using different number of terms in the Taylor series expansion. Vajari and Imam $[16,17]$ investigated the axial and torsional vibration of single-walled carbon nanotubes (CNTs) using DM. Axial vibration and wave propagation of a nanorod embedded in an elastic medium using DM has been discussed in [18]. Recently, dynamic and static analyses of CNTs have been examined via DM theory in $[19,20]$. The best agreement was obtained between DM model

\footnotetext{
* Corresponding author: metina@trakya.edu.tr
} 
and experiment for graphite in Ref. [20]. Considering previous studies according to the best knowledge of the authors, there is no study related to wave reflection and transmission in stepped nanorods by using DM. In this paper, expressions for the propagation, reflection and transmission matrices are derived for waves in stepped nanorods by using DM and elementary rod theories. These reflection and transmission matrices can provide a concise and systematic approach to dynamic analysis of nanorods. The advantages and physical basis of DM model are discussed in detail.

\section{DM Model of Nanotubes}

Analysis in DM theory starts with arraying each atom of elastic material as a node. These atoms (nodes) are located at certain finite distances (bond length) which are taken as $0.1421 \mathrm{~nm}$ for CNTS. Any two nodes are called as doublet and distance between them is called "doublet distance" of the considered material. Each node in the material can be elongate and/or rotate with respect to reference node. In this theory, elongation micro-stress $p_{\alpha}$, shear micro-stress $t_{\alpha}$ and torsional micro-stress $m_{\alpha}$ (Fig. 1) are considered in the elastic deformation. It is noted that these micro stresses are vector quantities. A symbolic deformation model for the DM theory is presented in Fig. 2. Each node $\alpha$ and their neighboring nodes are separated by doublet distance $\eta=\left|\xi_{\alpha}\right|$.

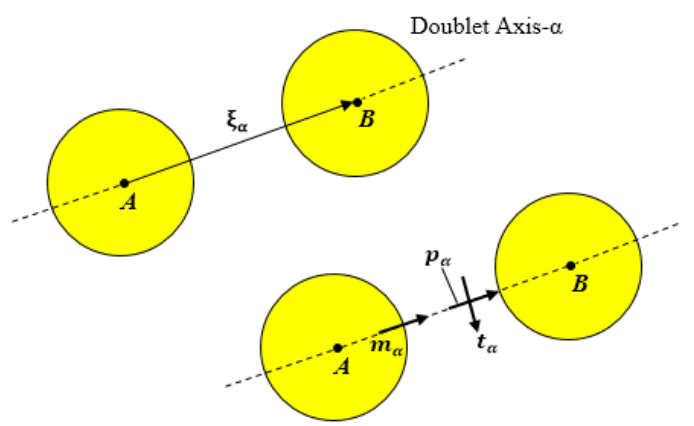

Fig. 1. Micro stresses in a doublet geometry [15]

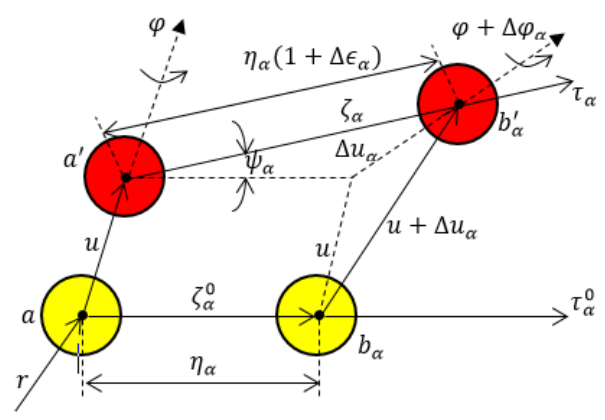

Fig. 2. Deformation of the doublet nodes [15]

After deformation of the nodes in a solid shown as $a_{\mathscr{C}}^{b}$ and $b_{\alpha}^{p}$. The increment of displacement is [15]:

$$
\Delta u_{\alpha}=\vec{u}\left(\vec{r}+\vec{\xi}_{\alpha}^{0}, t\right)-\vec{u}(\vec{r}, t)
$$

Here, $\vec{r}$ is the position vector of node $\alpha, t$ is the time and $\alpha=1,2, \ldots m, \quad m$ is the number of doublets. Elongation micro strain can be defined as [15]:

$$
\epsilon_{\alpha}=\frac{\vec{\tau}_{\alpha}^{0} \Delta \vec{u}_{\alpha}}{\eta_{\alpha}}
$$

where $\eta_{\alpha}$ is the doublet separation distance and $\vec{t}_{\alpha}^{\circ}$ is the unit vector in the $\alpha$-direction. Then, micro strain $\epsilon_{\alpha}$ can be expanded in a Taylor series as follows:

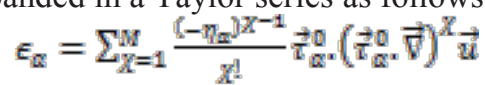

where Del operator and the number of the terms in the Taylor series expansion are denoted by $\vec{\nabla}$ and $M$, respectively. It should be noted that if $M$ equals to 1 it gives the classical elasticity theory equations. For the present study we considered the $\mathrm{M}=3$ in $\mathrm{DM}$ theory. The elongation micro stress is defined as [15]:

$$
p_{\alpha}=\Sigma_{\beta} B_{\alpha \beta} \epsilon_{\beta}
$$

where $p_{Q}$ is the elongation micro stress in the $\alpha$ doublet and $\epsilon_{\beta}$ is the axial micro strain in $\beta$ doublet and $B_{\alpha \beta}$ is the tension micro modulus between nodes $\alpha$ and $\beta$. The micro stress is linked to macro stress as below [15]:

$$
\sigma=\sum_{\alpha=1}^{n} \vec{\tau}_{\alpha}^{0} \vec{\tau}_{\alpha}^{0} \sum_{\chi=1}^{M} \frac{\left(-\eta_{\alpha}\right)^{\chi-1}}{\chi !}\left(\vec{\tau}_{\alpha}^{0} \cdot \vec{\nabla}\right)^{\chi-1} p_{\alpha}
$$

The expressions presented above is given for 3-D formulations. In the remaining part of the study plane stress assumption will be considered. The macro stressmicro stress relation can be written with using transformation matrix $[\mathrm{H}]$ in the following form:

$$
\{\sigma\}=[H]\{p\}
$$

By assumption of plane stress condition $\sigma, \varepsilon$ and $\mathrm{H}$ are

$$
\{\sigma\}=\left[\begin{array}{c}
\sigma_{x x} \\
\sigma_{y y} \\
\sigma_{x y}
\end{array}\right],[H]=\left[\begin{array}{lll}
\left(\tau_{11}^{0}\right)^{2} & \left(\tau_{21}^{0}\right)^{2} & \left(\tau_{31}^{0}\right)^{2} \\
\left(\tau_{12}^{0}\right)^{2} & \left(\tau_{22}^{0}\right)^{2} & \left(\tau_{32}^{0}\right)^{2} \\
\tau_{11}^{0} \tau_{12}^{0} & \tau_{21}^{0} \tau_{22}^{0} & \tau_{31}^{0} \tau_{32}^{0}
\end{array}\right],\{p\}=\left[\begin{array}{c}
p_{1} \\
p_{2} \\
p_{3}
\end{array}\right] \text { (7) }
$$

where $\vec{\tau}_{i j}^{0}$ are the cosines of the angles between the micro-stresses and Cartesian coordinates. Relation between micro strain and macro strain is:

$$
\{\dot{o}\}=[H]\{\varepsilon\}
$$

where

$$
\{\varepsilon\}=\left[\begin{array}{l}
\varepsilon_{x x} \\
\varepsilon_{y y} \\
\varepsilon_{x y}
\end{array}\right], \quad\{\epsilon\}=\left[\begin{array}{l}
\epsilon_{1} \\
\epsilon_{2} \\
\epsilon_{2}
\end{array}\right]
$$

The Hooke's law for the macro level is defined as

$$
\{\sigma\}=[C]\{\varepsilon\}
$$

where $C$ denotes the matrix of rigidities which incorporates the Lamé's constants $(\lambda, \mu)$ :

$$
C=\left[\begin{array}{ccc}
4 \mu\left(\frac{\lambda+\mu}{\lambda+2 \mu}\right) & \frac{2 \mu \lambda}{\lambda+2 \mu} & 0 \\
\frac{2 \mu \lambda}{\lambda+2 \mu} & 4 \mu\left(\frac{\lambda+\mu}{\lambda+2 \mu}\right) & 0 \\
0 & 0 & \mu
\end{array}\right]
$$

Using Eqs. (6), (8) and (10), following expression can be written as

$$
[C]=[H][B][H]^{T}
$$

where $\mathrm{B}$ is 


$$
B=\left[\begin{array}{lll}
\frac{4}{9} \mu \frac{7 \lambda+10 \mu}{\lambda+2 \mu} & \frac{4}{9} \mu \frac{\lambda-2 \mu}{\lambda+2 \mu} & \frac{4}{9} \mu \frac{\lambda-2 \mu}{\lambda+2 \mu} \\
\frac{4}{9} \mu \frac{\lambda-2 \mu}{\lambda+2 \mu} & \frac{4}{9} \mu \frac{7 \lambda+10 \mu}{\lambda+2 \mu} & \frac{4}{9} \mu \frac{\lambda-2 \mu}{\lambda+2 \mu} \\
\frac{4}{9} \mu \frac{\lambda-2 \mu}{\lambda+2 \mu} & \frac{4}{2} \mu \frac{\lambda-2 \mu}{\lambda+2 \mu} & \frac{4}{9} \mu \frac{7 \lambda+10 \mu}{\lambda+2 \mu}
\end{array}\right]
$$

For simplicity $\lambda=2 \mu$ may be chosen and from Eq. (13), it yields $B_{1 \mathrm{a}}=0$ which leads to

$$
B_{0}=\frac{8 \mu}{3}=E \text {. }
$$

A zigzag model of nanotube is shown in Fig. 3. For the present problem a node of $\alpha$ has three neighboring nodes. Their orientation can be given in terms of direction cosines: $\tau 1(1,0,0), \tau 2(-1 / 2, \sqrt{3} / 2,0), \tau 3(-1 / 2$, $\sqrt{3} / 2,0$ ) (Fig. 3). It is noted that by changing chiral angle of the doublet axis, the other models of nanotube can be obtained such as chiral and armchair models.

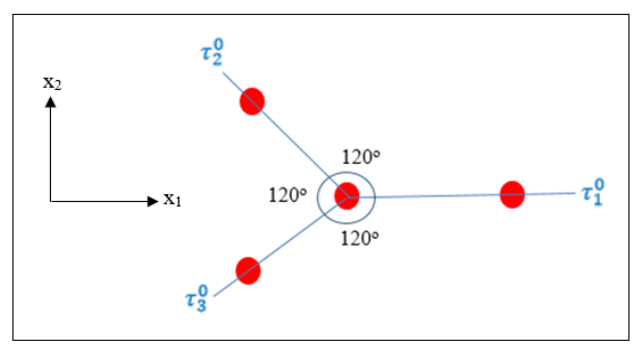

Fig. 3. Arrangement of atoms in a zigzag nanotube

The elongational micro strains (Eq. (3)) can be expanded in a Taylor series for the first three terms $(\mathrm{M}=3)$ by

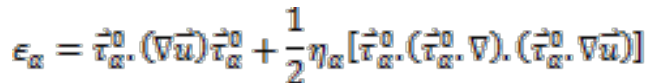

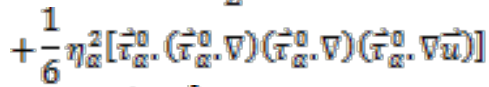

in Cartesian coordinate $\vec{\nabla}=\frac{\partial}{\partial x_{i}} \vec{e}_{i}$ and expanding $\vec{\tau}_{\tilde{\alpha}}^{0}$

$$
\begin{aligned}
\grave{o}_{\alpha}= & \tau_{\alpha m}^{0} \tau_{\alpha n}^{0}\left(\varepsilon_{m n}+\frac{1}{2} \eta_{\alpha} \tau_{\alpha s}^{0} \frac{\partial \varepsilon_{m n}}{\partial x_{s}}\right. \\
& +\frac{1}{6} \eta_{\alpha}^{2} \tau_{\alpha t}^{0} \tau_{\alpha s}^{0} \frac{\partial^{2} \varepsilon_{m n}}{\partial x_{t} \partial x_{s}}
\end{aligned}
$$

Similarly, the relation between macro stress and micro stress can be written as:

$$
\begin{aligned}
\sigma_{i j}^{(M)}= & \sum_{\alpha=1}^{n} \tau_{\alpha i}^{0} \tau_{\alpha j}^{0}\left\{\left(p_{\alpha}-\frac{1}{2} \eta_{\alpha} \vec{\tau}_{\alpha}^{0} \cdot\left(\nabla p_{\alpha}\right)\right.\right. \\
& \left.\left.+\frac{1}{6} \eta_{\alpha}^{2}\left[\left(\vec{\tau}_{\alpha}^{0} \cdot \nabla\right)\left(\vec{\tau}_{\alpha}^{0} . \nabla p_{\alpha}\right)\right]\right)\right\}
\end{aligned}
$$

The elongational micro stress can be defined as

$$
\begin{aligned}
p_{\alpha}= & B_{0} \tau_{\alpha m}^{0} \tau_{\alpha n}^{0}\left(\varepsilon_{m n}+\frac{1}{2} \eta_{\alpha} \tau_{\alpha s}^{0} \frac{\partial \varepsilon_{m n}}{\partial x_{s}}\right. \\
& +\frac{1}{6} \eta_{\alpha}^{2} \tau_{\alpha t}^{0} \tau_{\alpha s}^{0} \frac{\partial^{2} \varepsilon_{m n}}{\partial x_{t} \partial x_{s}}
\end{aligned}
$$

Then, using Eqs. (17) and (18), the relation between macro stress and macro strain is:

$$
\begin{aligned}
\sigma_{i j}^{(M)}= & \sum_{\alpha=1}^{n} B_{0} \tau_{\alpha i}^{0} \tau_{\alpha j}^{0} \tau_{\alpha m}^{0} \tau_{\alpha n}^{0}\left(\varepsilon_{m n}\right. \\
& \left.+\frac{1}{12} \eta_{\alpha}^{2} \tau_{\alpha t}^{0} \tau_{\alpha s}^{0} \frac{\partial^{2} \varepsilon_{m n}}{\partial x_{t} x_{s}}\right)
\end{aligned}
$$

Under the plane stress condition $-\frac{\varepsilon_{33}}{\varepsilon_{11}}=-\frac{\varepsilon_{22}}{\varepsilon_{11}}=v=\frac{1}{a}$ and considering only the axial strain, stress equation for DM theory can be obtained as

$$
\sigma_{x x}^{M}=B_{0}\left(\varepsilon_{11}+\frac{1}{12} \eta^{2} \frac{\partial^{2} \varepsilon_{11}}{\partial x^{2}}\right)
$$

For plane stress condition, $B_{0}=E$ in Eq. (14) in the nanotube. The first term in Eq. (20) is classical Cauchy Stress and the second term is doublet stress with a scale effect. It should be noted that doublet term depends on the number of Taylor series expansion in the solid.

\section{Governing Equations of a Nanorod}

Equations of motion and possible boundary conditions for a nanorod can be obtained by using Hamilton principle:

$$
\delta\left[\int_{t_{2}}^{t_{1}}(T-W-U) d t\right]=0
$$

where $\delta$ is the variational symbol, $U$ is the strain energy of the nanorod, $T$ is the kinetic energy and $W$ is the total work of external forces. The first variation of strain energy is

$$
\begin{gathered}
\delta U=-\int\left[A E\left(u^{u s}+s^{2} u^{I V}\right)\right]+\left[A E\left(u^{s}+s^{2} u^{u v}\right) \delta u\right]_{0}^{L} \\
+\left[A E\left(s^{2} u^{u v}\right) \delta u^{s}\right]_{0}^{L}
\end{gathered}
$$

where $s^{2}=\frac{\eta^{2}}{12}$.

where $A$ is the cross sectional area of the rod, $u$ is the axial displacement and $E$ is the elasticity modulus of the nanorod. The first variation of the kinetic energy is

$$
\delta T=\delta\left[\frac{1}{2} \int_{0}^{L} \rho u^{2} d x\right]
$$

where $\rho$ denotes the mass density of the nanorod and dot () defines the time derivative. The first variation of total work done by external forces (concentrated force P, distributed force $\mathrm{f}$ and double forces $\mathrm{R}$ ) can be written as:

$$
\begin{gathered}
\int_{t_{0}}^{t_{1}} \delta W d t=\int_{t_{0}}^{t_{1}} \int_{0}^{L} f(x, t) \delta u d x d t \\
+\int_{t_{0}}^{t_{1}}\left\{[P \delta u]_{0}^{L}+\left[R \delta u^{s}\right]_{0}^{L}\right\} d t
\end{gathered}
$$

The variational equation (Eq. 21) takes the following form:

$$
\begin{aligned}
& \int_{\mathrm{t}_{0}}^{\mathrm{t}_{1}} \int_{0}^{\mathrm{L}}\left[\operatorname{AE}\left(\mathrm{u}^{n s}+\mathrm{s}^{2} \mathrm{u}^{\mathrm{WV}}\right)+\mathrm{f}-\mathrm{p} \mathrm{u}\right] \delta \mathrm{u} d x d t \\
& \quad+\int_{\mathrm{t}_{0}}^{\mathrm{t}_{1}}\left[\left\{\mathrm{P}-\mathrm{AE}\left(\mathrm{u}^{s}+\mathrm{s}^{2} \mathrm{u}^{r s t}\right)\right\} \delta \mathrm{u}\right]_{0}^{\mathrm{L}} \mathrm{dt}
\end{aligned}
$$




$$
\begin{aligned}
& +\int_{t_{0}}^{t_{1}}\left[\left\{R-A E\left(-s^{2} u^{u s}\right)\right\} \delta u^{s}\right]_{0}^{L} d t \\
& +\int_{0}^{L}[p u ́ \delta u]_{t_{0}}^{t_{1}} d x=0
\end{aligned}
$$

Each term of the variational equation (25) must be zero. Thus, the equation of motion of the nanorod is obtained as

$$
A E\left[\left(u^{t s}(x, t)+s^{2} u^{I V}(x, t)\right]+f=p u(x, t)\right.
$$

The initial conditions satisfy the equation:

$$
\dot{u}\left(x, t_{1}\right) \delta u\left(x, t_{1}\right)-\dot{u}\left(x, t_{0}\right) \delta u\left(x, t_{0}\right)=0
$$

and the boundary conditions are:

$$
\begin{aligned}
& {\left[\mathrm{P}(\mathrm{L}, \mathrm{t})-\operatorname{AE}\left[\mathrm{u}^{s}(\mathrm{~L}, \mathrm{t})+\mathrm{s}^{2} \mathrm{u}^{t s s}(\mathrm{~L}, \mathrm{t})\right]\right] \delta \mathrm{u}(\mathrm{L}, \mathrm{t})} \\
& -\left[\mathrm{P}(0, \mathrm{t})-\operatorname{AE}\left[\mathrm{u}^{s}(0, \mathrm{t})+\mathrm{s}^{2} \mathrm{u}^{t s t}(0, \mathrm{t})\right]\right] \delta \mathrm{u}(0, \mathrm{t})=0, \\
& \quad\left[\mathrm{R}(\mathrm{L}, \mathrm{t})-\mathrm{AE}\left[-\mathrm{s}^{2} \mathrm{u}^{s t}(\mathrm{~L}, \mathrm{t})\right]\right] \delta u^{s}(\mathrm{~L}, \mathrm{t}) \\
& -\left[\mathrm{R}(0, \mathrm{t})-\mathrm{AE}\left[-\mathrm{s}^{2} \mathrm{u}^{s t}(0, \mathrm{t})\right]\right] \delta u^{s}(0, \mathrm{t})=0
\end{aligned}
$$

where the classical boundary conditions are displacement $u$ and the axial force $\mathrm{P}=A E\left(u^{b}+s^{2} u^{t s 5}\right)$. The boundary strain $u^{s}$ or the boundary double force $A E s^{2} u^{t s}$ are defined as the non-classical boundary conditions of the present DM theory. If by setting $s^{2}=0$ in Eq. (26), classical elasticity equation of motion is obtained. $s^{2}$ denotes the length scale parameter which is defined in terms of doublet separation distance.

\section{Wave Reflection and Transmission in Stepped Nanorods}

This section considers the wave reflection and transmission in a stepped nanorod (Fig. 4). It is seen that the neutral axes of the nanorods coincide.

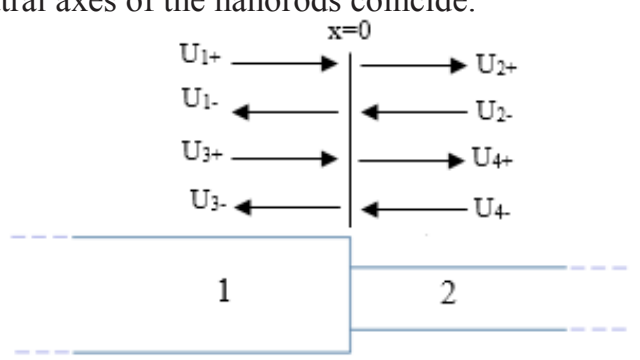

Fig. 4. Wave propagation in a stepped nanorod

Based on Eq. (26), equations of motions of a stepped nanorod can be expressed as:

$$
E A_{1} u_{1}^{s g}(x, t)+s^{2} E A_{1} u_{1}^{I V}(x, t)-\rho A_{1} \vec{u}_{1}(x, t)=0,
$$

$E A_{2} u_{2}^{s g}(x, t)+s^{2} E A_{2} u_{2}^{I V}(x, t)-\rho A_{2} u_{2}(x, t)=0 .(29)$

Denoting the axial displacements of the nanorod on the right and left sides of the neutral axis $(\mathrm{x}=0)$, the solution can be written by assuming the time-harmonic motion in the following form:

$$
\begin{aligned}
u_{1}(x, t)= & U_{1+} e^{i\left(\omega t-k_{1} x\right)}+U_{1-} e^{i\left(\omega t+k_{1} x\right)} \\
& +U_{3+} e^{i\left(\omega t-k_{2} x\right)}+U_{3-} e^{i\left(\omega t+k_{2} x\right)}, \\
u_{2}(x, t)= & U_{2+} e^{i\left(\omega t-k_{1} x\right)}+U_{2-} e^{i\left(\omega t+k_{1} x\right)} \\
& +U_{4+} e^{i\left(\omega t-k_{2} x\right)}+U_{4-} e^{i\left(\omega t+k_{2} x\right)} .
\end{aligned}
$$

where the eight amplitudes are related to reflection $\left(U_{1-,}, U_{2-}, U_{2-}, U_{4-}\right)$ and transmission
$\left(U_{1+}, U_{a+}, U_{2+,}, U_{4+}\right)$ waves. It is seen that depending on the frequency there are four propagating waves (two positive going and two negative going) for each crosssection of the stepped nanorod. Here, the wavenumbers are

$$
\begin{gathered}
k_{1}= \pm \sqrt{E-\sqrt{E\left(E-4 \rho \omega^{2} s^{2}\right)} / 2 E s^{2} x} \\
k_{2}= \pm \sqrt{E+\sqrt{E\left(E-4 \rho \omega^{2} s^{2}\right)} / 2 E s^{2}} .
\end{gathered}
$$

These wavenumbers can be obtained by assuming harmonic motion in Eq. (29). Eq. (29) gives a polynomial in $\mathrm{k}^{2}$. So the waves are dispersive in Eq. (31). The relation between the amplitudes and reflection and transmission coefficients can be explicitly defined as

$$
\begin{aligned}
& U_{1-}=r_{1} U_{1+}+t_{2} U_{2-} \\
& U_{2+}=t_{1} U_{1+}+r_{2} U_{2-} \\
& U_{a-}=r_{a} U_{a+}+t_{4} U_{4-} \\
& U_{4+}=t_{a} U_{a+}+r_{4} U_{4-}
\end{aligned}
$$

where $r_{1}, r_{2}, r_{2}, r_{4}$ are reflection coefficients and $t_{1}, t_{2}, t_{2}, t_{4}$ are transmission coefficients. When an incident wave with amplitudes of $U_{1+}, U_{a+}$ comes into rod-1, they produce reflected waves in rod-1 with amplitudes $r_{1} U_{1+}, r_{2} U_{\mathrm{a}+}$ and transmitted into rod-2 with amplitudes $t_{1} U_{1+}, t_{2} U_{a+\text {. }}$. If there is an incident wave with amplitudes $U_{2-}, U_{4-}$, in rod-2, reflected waves become $r_{2} U_{2-}, r_{4} U_{4-}$ and transmitted waves become $t_{2} U_{2-}, t_{4} U_{4-}$. To compute the reflection and transmission coefficients, idealized transition conditions can be defined as

$$
\begin{gathered}
u_{1}(0, t)=u_{2}(0, t) x_{x} \\
A_{1} E\left[u_{1}^{\prime}(0, t)+s^{2} u_{1}^{\prime \prime}(0, t)\right] \\
=A_{2} E\left[u_{2}^{\prime}(0, t)+s^{2} u_{2}^{\prime \prime}(0, t)\right], \\
A_{1} E s^{2} u_{1}^{s t}(0, t)=A_{2} E s^{2} u_{2}^{s t}(0, t), \\
u_{1}^{g}(0, t)=u_{2}^{s}(0, t) .
\end{gathered}
$$

By using Eqs. (32) and (33), values of the coefficients $r_{1}, r_{2}, r_{2}, r_{4}, t_{1}, t_{2}, t_{2}, t_{4}$ can be computed. Two conditions can be considered to compute the coefficients. Firstly, for $U_{1+}=U_{1+}=1$ and $U_{2-}=U_{4-}=0$, the amplitudes become $U_{1-}=r_{1}, U_{a-}=r_{a}, U_{2+}=t_{1}, U_{4+}=t_{a}$. Thus, the reflection and transmission matrices can be obtained as

$$
\begin{aligned}
& {\left[\begin{array}{cccc}
1 & 1 & -1 & -1 \\
k_{1} & k_{2} & k_{1} & k_{2} \\
-A_{1} E s^{2} k_{1}^{2} & -A_{1} E s^{2} k_{2}^{2} & A_{2} E s^{2} k_{1}^{2} & A_{2} E s^{2} k_{2}^{2} \\
S_{41} & S_{42} & S_{43} & S_{44}
\end{array}\right]\left[\begin{array}{c}
r_{1} \\
r_{3} \\
t_{1} \\
t_{3}
\end{array}\right]=} \\
& {\left[\begin{array}{c}
k_{1}+k_{2} \\
A_{1} E s^{2}\left(k_{1}^{2}+k_{2}^{2}\right) \\
A_{1} E\left(k_{1}+k_{2}\right)-A_{1} E s^{2}\left(k_{1}^{3}+k_{2}^{3}\right)
\end{array}\right]}
\end{aligned}
$$

where

$$
\begin{aligned}
& S_{41}=A_{1} E k_{1}-A_{1} E s^{2} k_{1}^{\mathrm{a}}, \\
& S_{42}=A_{1} E k_{2}-A_{1} E s^{2} k_{2 x}^{\mathrm{a}} \\
& S_{41}=A_{2} E k_{1}-A_{2} E s^{2} k_{1}^{\mathrm{a}}, \\
& S_{44}=A_{2} E k_{2}-A_{2} E s^{2} k_{2}^{\mathrm{a}} .
\end{aligned}
$$

By solving the linear equation system (34), coefficients $r_{1}, r_{2}, t_{1}, t_{2}$ can be easily obtained. 
Secondly, for $U_{1+}=V_{a+}=0$ and $U_{2-}=U_{4-}=1$, the amplitudes become $U_{1-}=t_{2}, U_{a-}=t_{4}, U_{2+}=r_{2}$, $U_{4+}=r_{4}$. Thus, the reflection and transmission matrices are

$$
\begin{aligned}
& {\left[\begin{array}{cccc}
1 & 1 & -1 & -1 \\
k_{1} & k_{2} & k_{1} & k_{2} \\
-A_{1} E s^{2} k_{1}^{2} & -A_{1} E s^{2} k_{2}^{2} & A_{2} E s^{2} k_{1}^{2} & A_{2} E s^{2} k_{2}^{2} \\
S_{41} & S_{42} & S_{43} & S_{44}
\end{array}\right]\left[\begin{array}{c}
t_{2} \\
t_{4} \\
r_{2} \\
r_{4}
\end{array}\right]=} \\
& {\left[\begin{array}{c}
-2 \\
k_{1}+k_{2}
\end{array}\right]} \\
& \left.A_{2} E\left(k_{1}+k_{2}\right)-A_{2} E s^{2}\left(k_{1}^{3}+k_{2}^{3}\right)\right]
\end{aligned}
$$

Similar to previous case, other four coefficients $r_{2}, r_{4}, t_{2}, t_{4}$ can be obtained by solving the linear system of equation (36).

\section{Numerical Results}

In this section, some typical reflection and transmission coefficients are presented for the classical elasticity and DM models in Fig. (5-7). The material properties of the nanorod are taken as $\rho=2300 \mathrm{~kg} / \mathrm{m}^{3}$ and $\mathrm{E}=1 \mathrm{TPa}$. The reflection and transmission coefficients are real for both theories. This corresponds to a single pair of propagating waves in a stepped nanorod. It is clearly seen that the reflection and transmission coefficients are independent from the frequency $\omega$ for the elementary rod theory (Fig. 5). Unlike the elementary rod theory, the reflection and transmission coefficients change with the frequency for the DM model (Figs. 6-7). The coefficients obtained from the two theories agree well with each other especially for low frequencies. The magnitude of the reflection and transmission coefficients increases with increasing the wave frequency for DM (especially when $\omega>10^{10}$ ) in Figs. (6-7). It should be noted that the difference of the coefficients are more apparent for higher frequencies especially for $\omega>10^{10}$. This is due to softening effect of the DM theory.

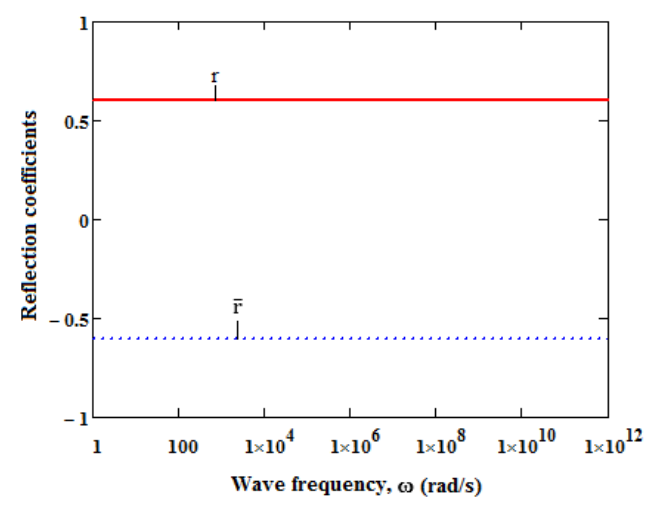

a)

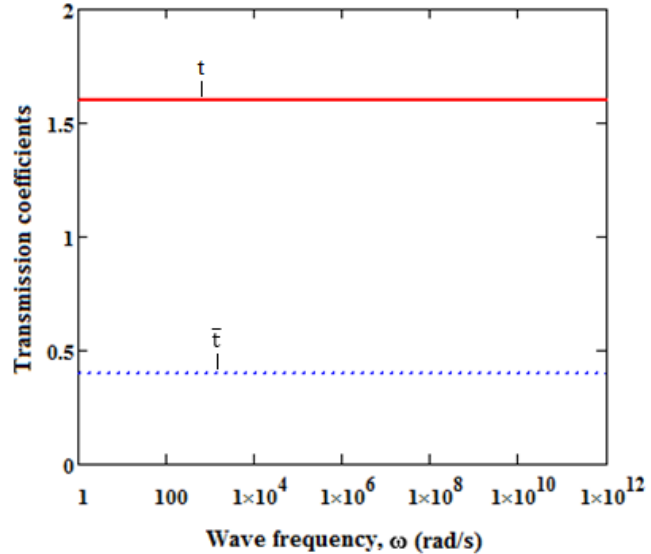

b)

Fig. 5. Reflection and transmission coefficients for classical elasticity theory $\left(\mathrm{A}_{2} / \mathrm{A}_{1}=0.25\right)$

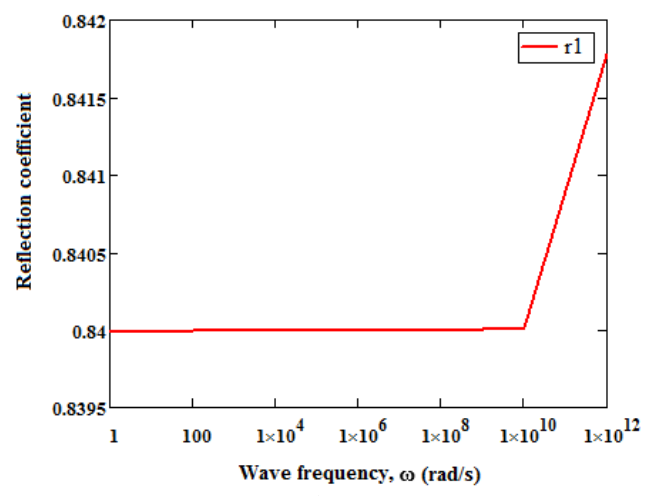

a)

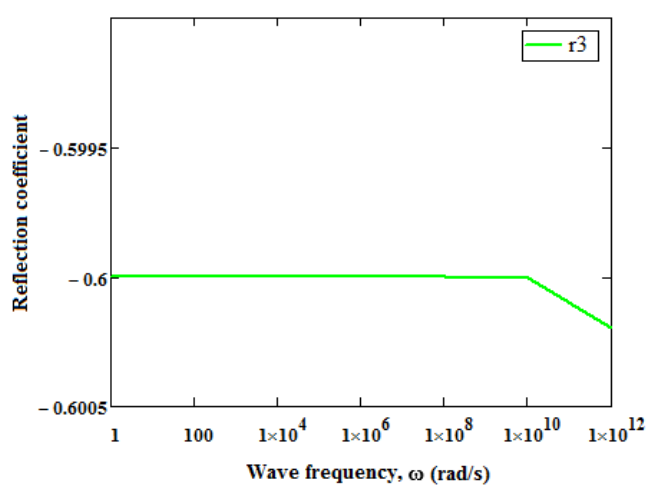

b)

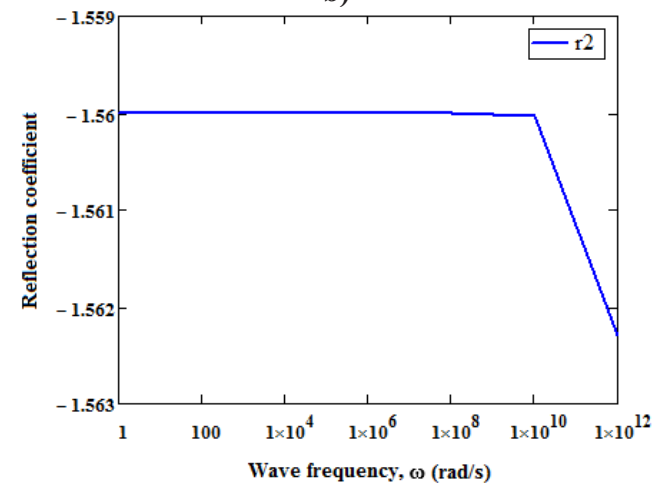

c) 


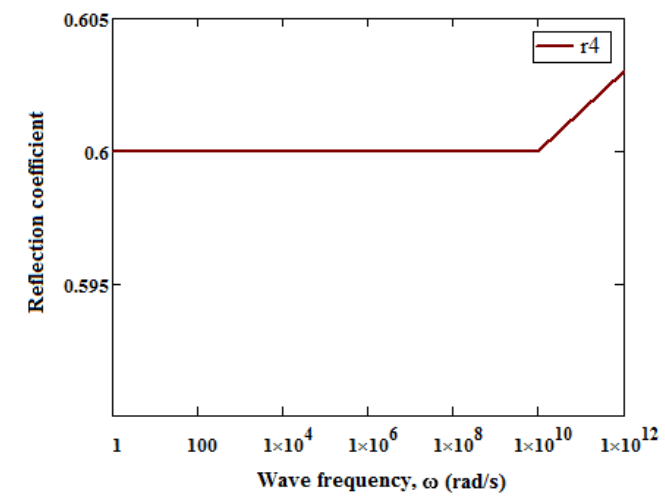

d)

Fig. 6. Reflection coefficients for DM theory $\left(\mathrm{A}_{2} / \mathrm{A}_{1}=0.25\right.$, $\eta=0.1421 \mathrm{~nm})$

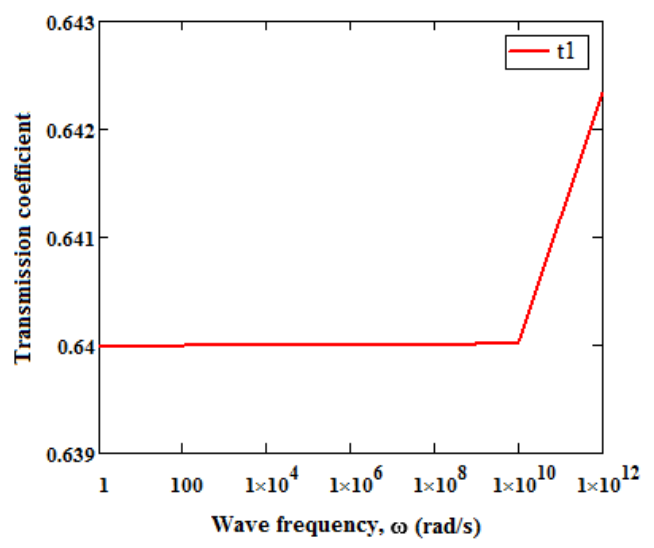

a)

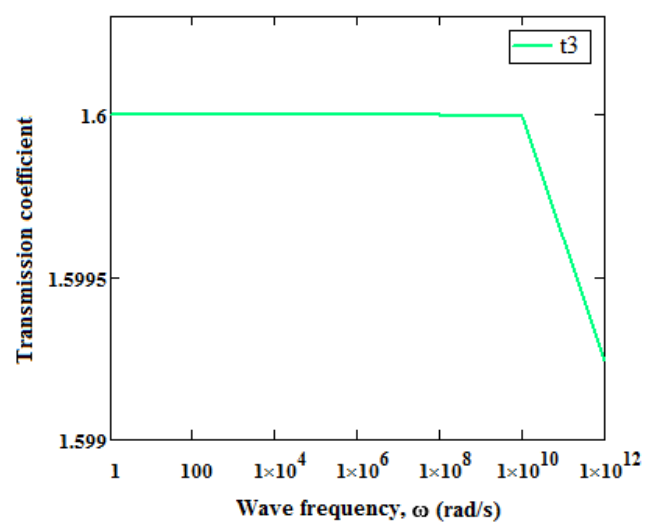

b)

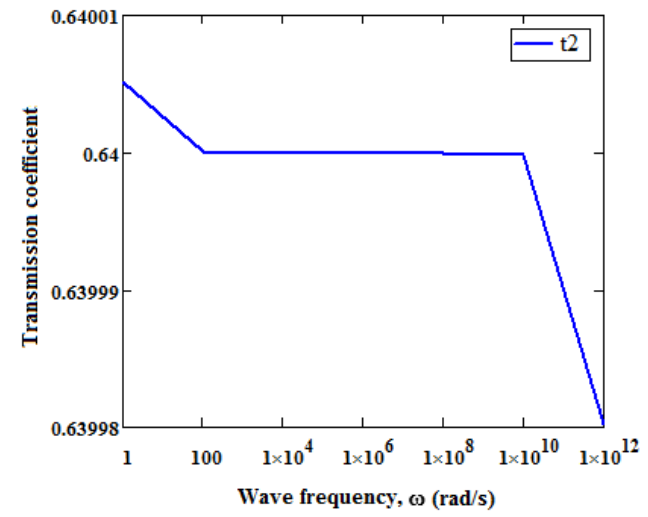

c)

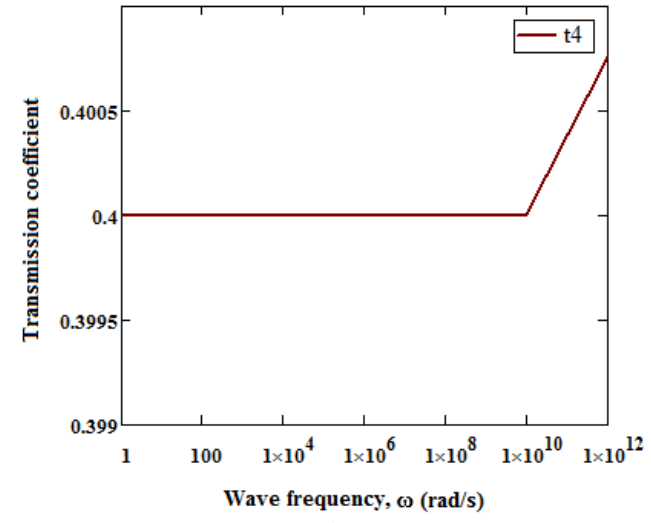

d)

Fig. 7. Transmission coefficients for DM theory $\left(A_{2} / A_{1}=0.25\right.$, $\eta=0.1421 \mathrm{~nm})$

\section{Conclusions}

In this study, DM theory was used to determine the wave propagation, reflection and transmission relations in the stepped nanorods. The vibration analysis of the nanorods become systematic and concise with the availability of propagation, reflection and transmission matrices. It was obtained that magnitudes of the coefficients are almost same for the low frequencies however there is an important difference for high frequencies for DM and classical theory. These results may be given for such discontinuities caused by general point supports in the future studies.

\section{References}

1. L. Cremer, M. Heckl, Structure borne Sound (Berlin: Springer-Verlag, 1988)

2. P. Hagedorn, W. Seemann, (VPI-Symposium, Blacksburg, 1995)

3. W. Seemann, J. Sound Vib. 197, 5 (1996)

4. B.R. Mace, J. Sound Vib. 97, (1984)

5. N.R. Harland, B.R. Mace, R.W. Jones, J. Sound Vib. 241, 5 (2001)

6. C. A. Tan, B. Kang, J. Sound Vib. 213, 3 (1998)

7. C. A. Tan, B. Kang, Int. J. Solids Struct. 36, (1999)

8. J.F. Doyle, Wave Propagation in Structures (Spring-Verlag, New York, 1989)

9. M.Y. Wang, H.L. Li, Y.L. Dong, G.P. Li, C.L. Zhong, J. Xu, ACES J. 31, (2016)

10. E. Cosserat, F. Cosserat, Sur la théorie des corps déformables (Herman,Paris, 1909)

11. R.D. Mindlin, Arch. Ration Mech. Anal. 16, (1964)

12. S. Kong, S. Zhou, Z. Nie, K. Wang, Int. J. Eng. Sci. 47, (2009) 
13. V.T. Granik, Technique report IM/MGU (Inst. of Mech. of Moscow State Univ., 1978)

14. V.T. Granik, M. Ferrari, Mech. Mater. 15, (1993)

15. M. Ferrari, V.T. Granik, A. Imam, J. Nadeau, Advances in Doublet Mechanics (Springer, 1997)

16. A.F. Vajari, A. Imam, Indian J. Phys. 90, 4 (2016)

17. A.F. Vajari, A. Imam, ZAMMP (to be published)

18. U. Gul, M. Aydogdu, G. Gaygusuzoglu, Comp. Struct. 160, (2017)

19. U. Gul, M. Aydogdu, J. Engng. Math. (to be published)

20. U. Gul, M. Aydogdu, Physica E 93, (2017) 